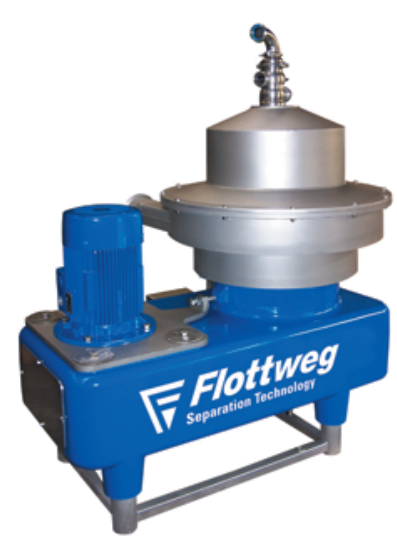

Separator der AC-Baureihe von Flottweg

konsequent servicefreundlich konstruiert, ist dieser Dekanter für den langjährigen harten Einsatz bei geringstem Wartungsaufwand konzipiert.

Neuentwicklungen von Flottweg sind die Separatoren der AC-Baureihe. Sie sind mit dem SoftShot-Entleerungssystem ausgerüstet und zeichnen sich durch besonders exakte Teilentleerungen und ruhigen Lauf aus. Separatoren mit selbstentleerender Trommel werden zur Abscheidung von suspendierten Feststoffen aus Flüssigkeiten und zur Trennung von zwei nicht ineinander löslichen Flüssigkeiten bei gleichzeitiger Abscheidung von Feststoffen eingesetzt. Zu den Einsatzgebieten der Flottweg AC-Separatoren gehören:

- Raffinerien: Reinigung von Slopöl

- Mechanische Metallverarbeitung: Pflege von Kühlschmieremulsion

- Walzwerke: Reinigung von Schmieröl

- Aufbereitung von Rohöl: Entsalzung

- Keramik und Glasverarbeitung: Klärung von $\mathrm{Ab}$ wasser.

Informationen:

Flottweg GmbH \& Co. KGaA

Industriestraße 6-8

D-84137 Vilsbiburg

Tel. +49-8741-301-353

Fax +49-8741-301-303

mk@flottweg.com

www.flottweg.com
MASCHINEN-/VERFAHRENSTECHNIK

\section{Huber lieferte 15.000. Maschine aus}

Im September 2004 war es soweit: Die 15.000. HuberMaschine, ein ROTAMAT ${ }^{\circledR}$ Scheibeneindicker RoS2S, wartete auf die Auslieferung zur Kläranlage der Gemeinde Sölden in Tirol. Die ersten Huber-Maschinen wurden 1983 gefertigt und ausgeliefert, wobei es sich um den ROTAMAT ${ }^{\circledR}$. Feinrechen Rol handelte. Basierend auf dem ROTAMAT $^{\oplus}$-Prinzip „Sieben - Waschen - Transportieren Entwässern in einer Maschine" wurde über die Jahre die ROTAMAT ${ }^{\oplus}$-Familie entwickelt und erfolgreich in den weltweiten Markt der Abwasserreinigung eingeführt. In den letzten Jahren wurde die ROTAMAT ${ }^{\circledR}$ Familie durch den STEP SCREEN ${ }^{\circledR}$ und später noch durch die MAX-Famile er-
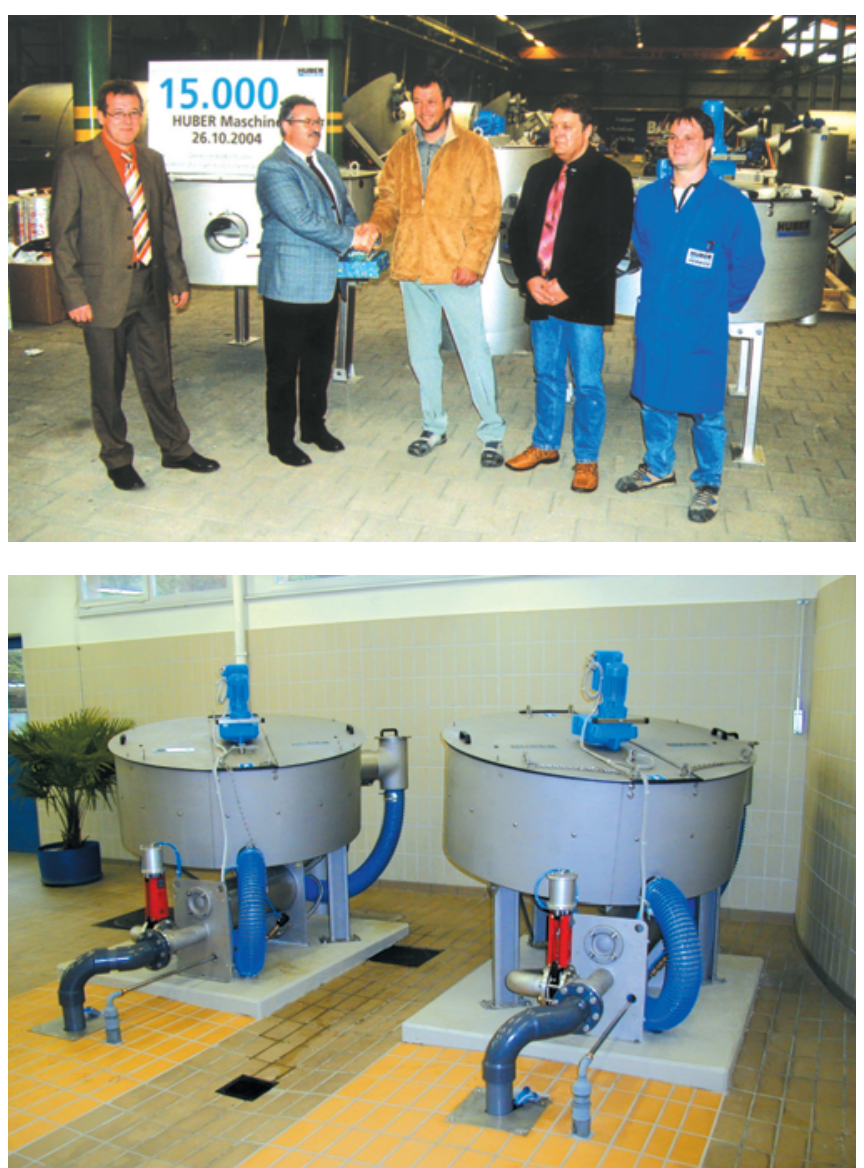

lebung (> $500 \mathrm{EW)}$ ) als auch in Form der „MembraneClearBox“ oder „HoneyComb“ für die dezentrale Abwassereinigung zur Verfügung.

15.000 Maschinen gefertigt und ausgeliefert, das ist eine Zahl, auf die man stolz sein kann. Aber mit der Fertigung und Auslieferung hört die Verantwortung von Huber nicht auf. Alle Maschinen müssen installiert, in Betrieb genommen und dann kontinuierlich gewartet werden. Die Wartung beinhaltet die Reparatur (vor Ort oder im Werk), die Versorgung der Betreiber mit Verschleiß- und Ersatzteilen sowie die Optimierung der Anlagen. Um diesen Anforderungen auch künftig Rechnung tragen zu können, wurde in den letzten Monaten eine neue Servicehalle errichtet und in Betrieb genommen. Diese Halle, ausgestattet mit verschiedensten Fertigungsmaschinen, gestattet es nun, Reparaturen schnellstmöglich auszuführen. Außerdem werden in dieser Servicehalle die zahlreichen Vorführanlagen gewartet und auf den nächsten Einsatz beim Kunden vorbereitet.

\section{Informationen: \\ Hans Huber AG Postfach 63 \\ D-92334 Berching \\ Tel. +49-8462-201-o \\ info@huber.de \\ www.huber.de}

\section{PHOTOMETER}

\section{DR 5000 UV-VIS von HACH LANGE}

Das DR 5000 UV-VIS Spektralphotometer stellt die neueste Entwicklung von HACH LANGE im Bereich Photometrie dar: hochpräzise Optik, intuitive

Menüführung, ergonomisches Design. Dabei ist es mehr und kann es mehr als ein „beliebiges“ UV-VIS- 